\title{
Análisis del componente inorgánico del calculo dental proveniente de individuos adultos mediante difracción de rayos $x$ y microscopia de fuerza atómica.
}

\section{Analysis of the inorganic component of dental calculus from adult through $x$-rays difraction and atomic force microscopy.}

\author{
L. Barrios $^{1}$ | M. Montero ${ }^{2}$ | F. Rojas-SÁnchez ${ }^{3}$ | T. BOnillo ${ }^{4}$ R. \\ VARGAS $^{5}$
}

Recibido: 7 de octubre de 2019/ Aceptado: 23 de abril de 2020

\footnotetext{
${ }^{1}$ Complejo Hemato - Oncológico y de radiocirugía, IVSS. Caracas (Venezuela). email: leonardoalberto@gmail.com

${ }^{2}$ Instituto de investigaciones odontológicas,

"Raúl Vincentelli", Facultad de odontología,

Universidad Central de Venezuela. email: maglymontero44@gmail.com

${ }^{3}$ Instituto de investigaciones odontológicas, "Raúl Vincentelli", Facultad de odontología, Universidad Central de Venezuela email: rojas.fatima@gmail.com

${ }^{4}$ Unidad de Estructura Molecular, Fundación IDEA.email: tbonillo@ hotmail.com

${ }^{5}$ Unidad de Estructura Molecular, Fundación IDEA.email: rvargas@idea.gov.ve
}

El cálculo dental es producto de la mineralización de la biopelícula dental, dentro de los distintos componentes que lo conforman se encuentran compuestos inorgánicos, una matriz orgánica compuesta de proteínas salivales selectivamente absorbidas del medio bucal y varias especies de microorganismos. El objetivo del presente trabajo fue determinar la composición inorgánica del cálculo dental empleando difracción de rayos X (DRX por sus siglas en ingles) y Microscopía de Fuerza Atómica (MFA por sus siglas en ingles), así como también el análisis por Microscopia Electrónica de Barrido - Espectroscopia de Rayos X por Energía Dispersiva (SEM - EDX por sus siglas en ingles). Para ello, se recolectaron muestras de cálculo dental supragingival (material de desecho durante actividades de tartrectomía), proveniente de 4 muestras de pacientes adultos, seleccionados de manera no aleatoria, que asistieron voluntariamente a la Facultad de Odontología de la UCV. Cada muestra fue almacenada en tubos demicrocentrífuga hasta su procesamiento. Los estudios de DRX y MFA, fueron realizados en la Unidad de Estructura Molecular, del Instituto de Estudios Avanzados (IDEA). El análisis por EDX fue realizado en el Centro de Microscopía Electrónica de la UCV. Los resultados indicaron que el componente inorgánico principal del cálculo dental es la hidroxiapatita en todas las muestras analizadas, tanto por difracción de rayos X como del análisis por EDX.

The dental calculus is a product of the mineralization of the dental biofilm; within the various components that comprise it are inorganic compounds, an organic matrix composed of salivary proteins selectively adsorbed from the oral environment and several species of microorganisms. The objective of this work was to determine the inorganic composition of dental calculus using X - ray Diffraction (XRD) and Atomic Force Microscopy (AFM), as well as the analysis by Scanning Electron Microscopy - Energy Dispersive X Ray Spectroscopy (SEM - EDX), this analysis was done at the Electron Microscopy Centre at the UCV. They collected samples of supragingival dental calculus (waste material during tartrectomy activities), from 4 samples of adult patients, selected in a non-random manner, who voluntarily attended the Faculty of Dentistry of the UCV. Each sample was stored in Eppendorf tubes until processing. The XRD and AFM studies were carried out in the Molecular Structure Unit of the Institute for Advanced Studies (IDEA). The results indicated

\footnotetext{
* Esta obra está bajo una licencia Creative Commons Reconocimiento - NoComercial 4.0 Internacional @() ()

* This work is licensed under a Creative Commons Attribution-NonCommercial 4.0 International License. () ()
} 
that the main inorganic component of dental calculus is hydroxyapatite in all samples analyzed, both by $\mathrm{X}$ ray diffraction and EDX analysis.

PALABRAS CLAVES

Cálculo dental, Difracción de Rayos X, Microscopía de Fuerza Atómica,

Hidroxiapatita.

KEYWORDS

Dental Calculus, X Ray Diffraction, Atomic Force Microscopy,

Hydroxyapatite.

\section{I | INTRODUCCIÓN}

I A placa dental es un tipo de biopelícula que se define como una comunidad microbiana diversa que Le encuentra en la superficie dental embebida en una matriz de polímeros de origen bacteriano y salival (A. Loera y Guerrero, 2012), que posee una alta diversidad de especies bacterianas y presenta una estructura ordenada, englobada en polisacáridos extracelulares, que le confiere resistencia ante las defensas del huésped y los antibióticos. Hayashizaki y colaboradores (J. Hayashizaki 2008), establecieron que la composición química de los cálculos dentales es variable, reflejándose en la cristalinidad del mismo; son necesarias más investigaciones para descubrir los factores relacionados a los sitios específicos de aparición dentro de una misma cavidad bucal y entre distintos individuos. Por su parte, D.J. White (White, 2000) menciona que la formación de cálculo dental ocurre en las cavidades bucales de casi todos los individuos a lo largo y ancho del mundo. Se sabe que las poblaciones en donde se tiene acceso a servicios profesionales de salud bucal y que tienen prácticas de higiene adecuadas, la formación de cálculos se restringe a las superficies dentales adyacentes a la salida de los conductos excretores de las glándulas salivales debido a que la saliva en un fluido sobresaturado en iones de calcio y fosfato de manera fisiológica (B. Anuradha, 2016).

A pesar de conocerse el mecanismo de crecimiento del cálculo dental, todavía no está claro por qué o cómo empieza el proceso de la calcificación de la biopelícula dental. Al respecto, existen diversas estudios que han tratado de explicar cómo se produce este fenómeno, aunque ninguna está definitivamente probada (S. R. Epstein, 1980). Algunos estudios señalan que los pacientes con elevada tendencia a formar cálculo dental poseen mayores niveles de proteínas, calcio y lípidos en saliva, pero no se ha establecido su papel en la formación del cálculo dental.(Dos Reis. 2016)

Para tratar de comprender más sobre este proceso, se ha revisado la formación del cálculo urinario; al respecto, se conoce que mucoproteínas atípicas forman una matriz de calcificación (S. R. Epstein, 1980) y estos hallazgos pueden reproducirse en el medio bucal,debido a que existen mucoproteínas como calcoprotectina y osteopontina, que podrían actuar como matriz de calcificación. La calcoprotectina derivadas de los granulocitos, macrófagos, saliva y epitelio oral (y M. E. González, 2016); y la osteonectina, glicoproteína ligada al calcio, son capaces de originar núcleos de calcificación, por lo que estos estudios sugieren que los pacientes que sufren de cálculo renal tienden a formar en mayor cantidad cálculo dental (A. J. Caballero, 2011).

Otro proceso similar es la sialolitiasis, descrita como una patología obstructiva de la glándula salival, caracterizada por la formación de cálculos dentro del parénquima glandular o en los conductos glandulares de mayor o menor calibre (A. J. Caballero, 2011).

Al igual que los cálculos dentales, estos están formados por dos tipos de componentes: un grupo 

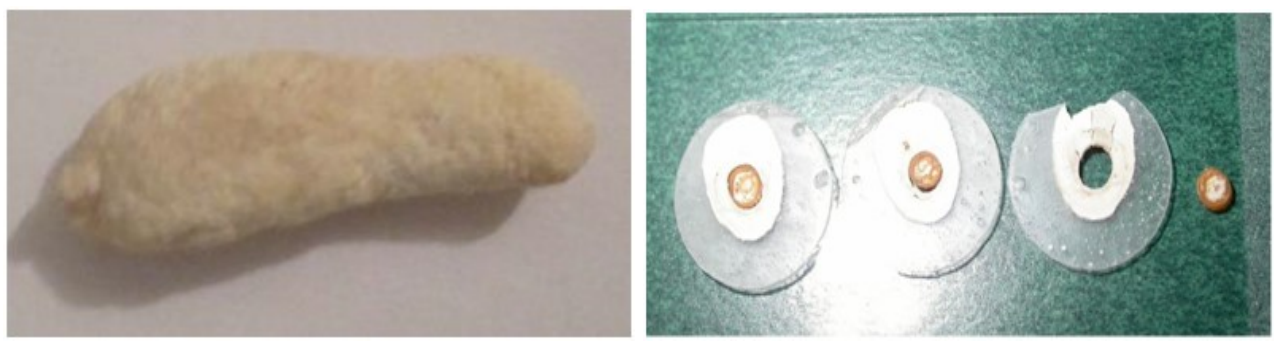

Figura 1: Sialolito extraído a paciente

de origen orgánico y otro inorgánico. Entre los principales constituyentes orgánicos se encuentran productos de la saliva (glucoproteínas y mucopolisacáridos), lípidos y detritus celulares, mientras que el componente inorgánico principal es el carbonato - apatita, acompañado de otras sales de calcio, además de diferentes tipos de fosfatos, magnesio, hierro, cobre y zinc (Cullity, 1959).

En función de todo lo anteriormente descrito, se planteó como objetivo del presente trabajo, determinar la composición inorgánica del cálculo dental empleando la difracción de rayos X, la microscopía de fuerza atómica y el análisis por EDX.

\section{METODOLOGÍA}

$E^{s}$ STE trabajo es experimental, se recolectaron muestras de cálculo dental supragingival (material de desecho durante actividades de tartrectomía), proveniente de 4 pacientes adultos, seleccionados de manera no aleatoria, que asistieron voluntariamente a la Facultad de Odontología de la UCV.

Una vez recuperadas, las muestras fueron colocadas en tubos de microcentrífuga y enviadas a la Unidad de Estructura Molecular del IDEA, para realizar el análisis por medio de la difracción de rayos $\mathrm{X}$. Todas fueron tratadas de la misma manera: se lavaron con agua miliq para remover restos de alimentos y sangre; posteriormente fueron colocadas en una estufa de desecación a una temperaturade $37^{\circ} \mathrm{C}$, durante un periodo de 24 horas, por último fueron pulverizadas en un mortero de agata y colocadas en los portamuestras de $\mathrm{Ni}$, momento en el cual se procedió a realizar los diferentes análisis.

Adicionalmente, se empleó un sialolito para comparar el componente inorgánico de ambos cálculos (salival y dental). El sialolito fue extraído quirúrgicamente por un cirujano maxilofacial y donado para realizar los diferentes estudios. Sus dimensiones fueron de: $39.7 \mathrm{~mm}$ de largo y 8.5 x $6.5 \mathrm{~mm}$ de grosor, con un peso de $4.48 \mathrm{~g}$ ( $\mathrm{fig}$ 1). El espécimen fue transportado en seco, fue incluido en acrílico transparente y cortado en un micrótomo de baja velocidad. Los discos obtenidos de aproximadamente $1 \mathrm{~mm}$ de espesor, fueron colocados directamente en el difractómetro y en el MFA para su análisis.

Por ultimó las muestras fueron enviadas al Centro de Microscopia Electrónica de la UCV, donde fueron analizadas usando la técnica de EDX con un microscopio de barrido marca JEOL JXA 8900R. Los parámetros usados para la realización de este análisis fueron: HV: $20 \mathrm{kV}$, tipo de detector: ETD, magnitud entre $200 \mathrm{X}$ y $5000 \mathrm{X}$, las muestras no requirieron de ningún tipo de preparación química. 


\begin{tabular}{lllllll}
\hline & Muestra & Peso $(\mathbf{k g})$ & Color externo & Superficie & Textura & Dureza \\
\hline FF45001 & 45.3 & $4.5 \times 3.3 \times 1.1$ & Marrón crema & Granular & Laminar & semiduro \\
F56001 & 1.8 & $3.2 \times 3.4 \times 0.8$ & Marrón & Granular & Laminar & Duro \\
M51001 & 92 & $9.8 \times 3.4 \times 0.1$ & Crema & Granular & Laminar & Duro \\
Sialolito & 4475.5 & $30 \times 8.5 \times 6.5$ & Crema & Granular & Laminar & Duro \\
\hline
\end{tabular}

Tabla 1: Características de las distintas muestras estudiadas.

\section{Análisis por Difracción de Rayos X:}

El análisis se realizó empleando un equipo de Difracción de rayos marca XD2 Phaser - BRUKER para muestras policristalinas, con las siguientes características:

- Voltaje acelerador de $30 \mathrm{kV}$, una corriente de 10 $\mathrm{mA}$.

- Geometría del montaje experimental: Bragg - Brentano.

- Reflexión: $\theta-\theta$

- Radiación: $\lambda_{\text {Cua }}=1.542 \dot{A}$
- Barrido: $10^{\circ}-60^{\circ}$ en $2 \theta$

- $\Delta 2 \theta=0.02^{\circ}$ (Paso del Barrido)

- $\tau=0.5^{\prime}$ (tiempo del paso del barrido)

- Temperatura y presión ambiental.

- Porta muestra de Ni con ranura lineal de $2 \mathrm{~mm}$ para el depósito de la muestra.

\section{Análisis por microscopía de fuerza atómica}

Las muestras fueron colocadas en los portamuestras respectivos para ser analizadas en el MFA, el análisis fue netamente morfológico. Para todas las muestras, se fijó la frecuencia en $0.3 \mathrm{KHz}$ con una resolución de $1024 \times 1024$ pixels en modo tapping.

\section{| RESULTADOS}

Se realizó un análisis cualitativo de las muestras, donde se determinó su peso, tamaño, así como también otras características morfológicas, estas se muestran en la tabla 1 .

\section{| DIFRACCIÓN DE RAYOS X}

Los resultados de las pruebas de Difracción de Rayos X (DRX), tanto para los cálculos dentales como para el sialolito, mostraron concordancia con los difracto-gramas del fosfato $\mathrm{Ca}_{5}\left(\mathrm{PO}_{4}\right)_{3}(\mathrm{OH})$ encontrados en la base de datos del Centro Internacional para la Data de Difracción (ICDD por sus siglas en inglés) y que se muestra en la figura (2), este fosfato es el principal responsable de la formación de cálculos en los dientes y en los conductos salivales. La correspondencia de los primeros picos en los difractogramas obtenidos de las muestras analizadas con los de la base de datos se muestra en las figuras (3) y (4).

Es importante acotar, que debido a que la cantidad de las muestras que fueron analizadas, no son lo suficientemente abundantes los picos de los difractogramas no tienen la suficiente intensidad y definición, aun considerando un tiempo de exposición de la muestra considerablemente extenso, de igual forma el pico más intenso y definido que se observa en todas los difractogramas corresponde al del portamuestra, que una vez que finalizado el barrido en la muestra el equipo sigue detectando hasta 


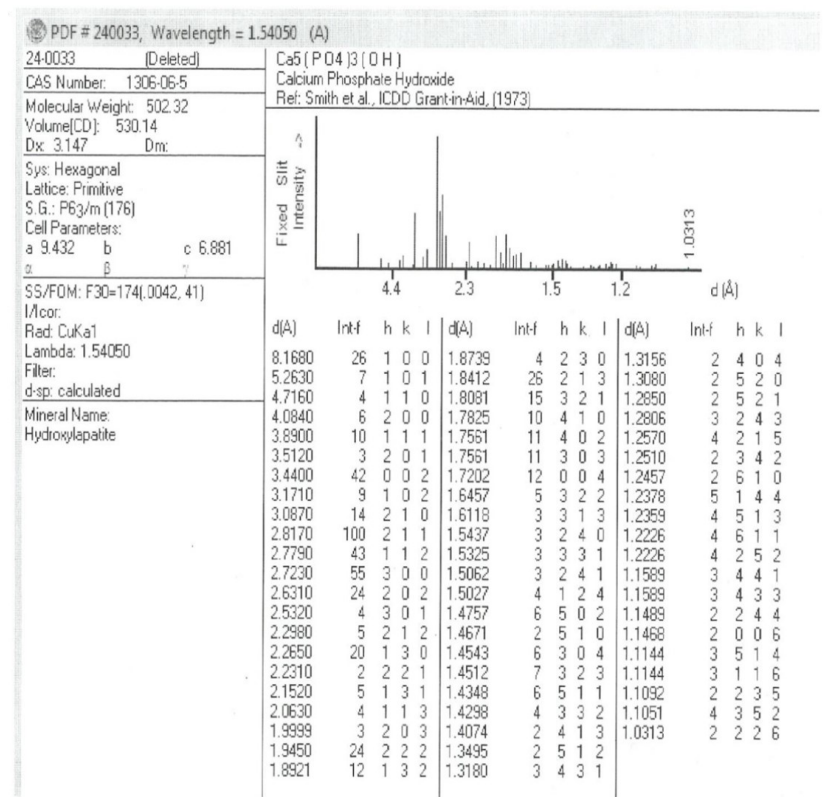

Figura 2: Difractograma de la Hidroxiapatita (Base de datos del ICDD).

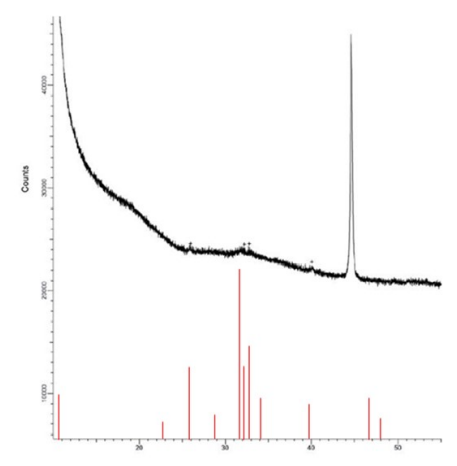

(a) Muestra F45001

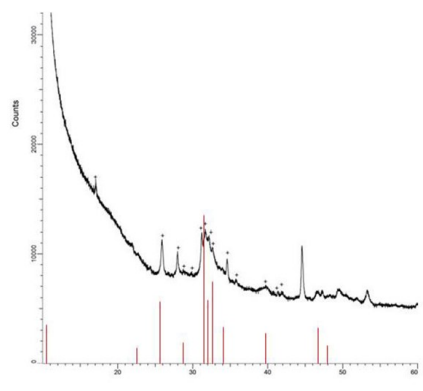

(b) Muestra F56001.

Figura 3: Difractogramas obtenidos de las muestras analizadas en un rango de 0 a 50 para el ángulo $2 \theta$.

que aparece este pico. Las líneas rojas mostradas en los difractogramas representan las posiciones de los picos de la Tabla de la base de datos del ICDD.

Para el cálculo de los parámetros se seleccionaron los picos con mayor intensidad que corresponden con los índices 211 y 002, y haciendo uso de (1) se determinaron los parámetros para cada muestra.

Como se describe en el capítulo 10 "The determination of Crystal Structure" del libro "Elements of X -Ray Diffraction" (Cullity, 1959). La expresión para calcular la distancia interreticular (d) de los planos hkl del sistema cristalino hexagonal está dado por: 


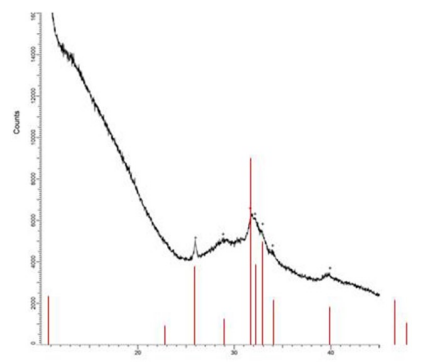

(a) Muestra F51001

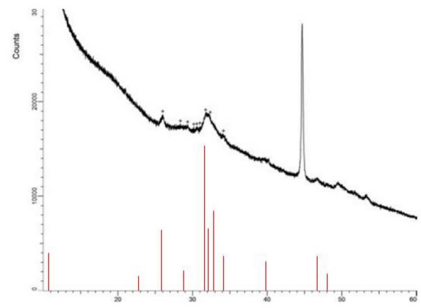

(b) Muestra SIA001.

Figura 4: Difractogramas obtenidos de las muestras analizadas en un rango de 0 a 50 para el ángulo $2 \theta$.

\begin{tabular}{l|lll|lll}
\hline & \multicolumn{3}{|c}{ Para los índices 221 } & \multicolumn{3}{c}{ Para los índices 002 } \\
\hline Muestra & $\theta$ & $d(\dot{A})$ & $a(\dot{A})$ & $\theta$ & $d(\dot{A})$ & $a(\dot{A})$ \\
\hline FF45001 & 15.60 & 2.867 & 9.623 & 12.86 & 3.460 & 6.919 \\
F56001 & 15.90 & 2.814 & 9.428 & 13.00 & 3.427 & 6.854 \\
M51001 & 15.75 & 2.840 & 9.519 & 12.90 & 3.453 & 6.906 \\
SIA001 & 16.50 & 2.714 & 9.017 & 12.90 & 3.453 & 6.906 \\
\hline
\end{tabular}

Tabla 2: Características de las distintas muestras estudiadas.

$$
\frac{1}{d^{2}}=\frac{\lambda^{2}}{4}\left[\frac{4}{3}\left(\frac{h^{2}+h k+k^{2}}{a^{2}}\right)+\frac{l^{2}}{c^{2}}\right]
$$

Donde a y c son los parámetros de celda, y de donde se deriva que la relación señal observada calculada es:

$$
\sin ^{2}(\theta)=\frac{\lambda^{2}}{4}\left[\frac{4}{3}\left(\frac{h^{2}+h k+k^{2}}{a^{2}}\right)+\frac{l^{2}}{c^{2}}\right]
$$

Siendo $\theta$ la posición angular del pico de difracción observado. Los parámetros de celda calculados del sistema estudiado son mostrados en la tabla 2 .

Los parámetros promedio calculados son:

$$
\bar{a}=9.347 \cdot A ; \quad \bar{c}=6.897 \cdot A
$$

Estos valores calculados son absolutos, debido a que son individuales de cada paciente analizado. El tamaño del cristal se calculó a partir de los difractogramas y haciendo uso de la ecuación de Scherer que está dada por:

$$
t(h k l)=\frac{0.9 \lambda}{B \cos \theta_{(h k l)}}
$$

Donde $\lambda$ es la longitud de onda de los rayos X, B es el ancho a media altura del pico de mayor 

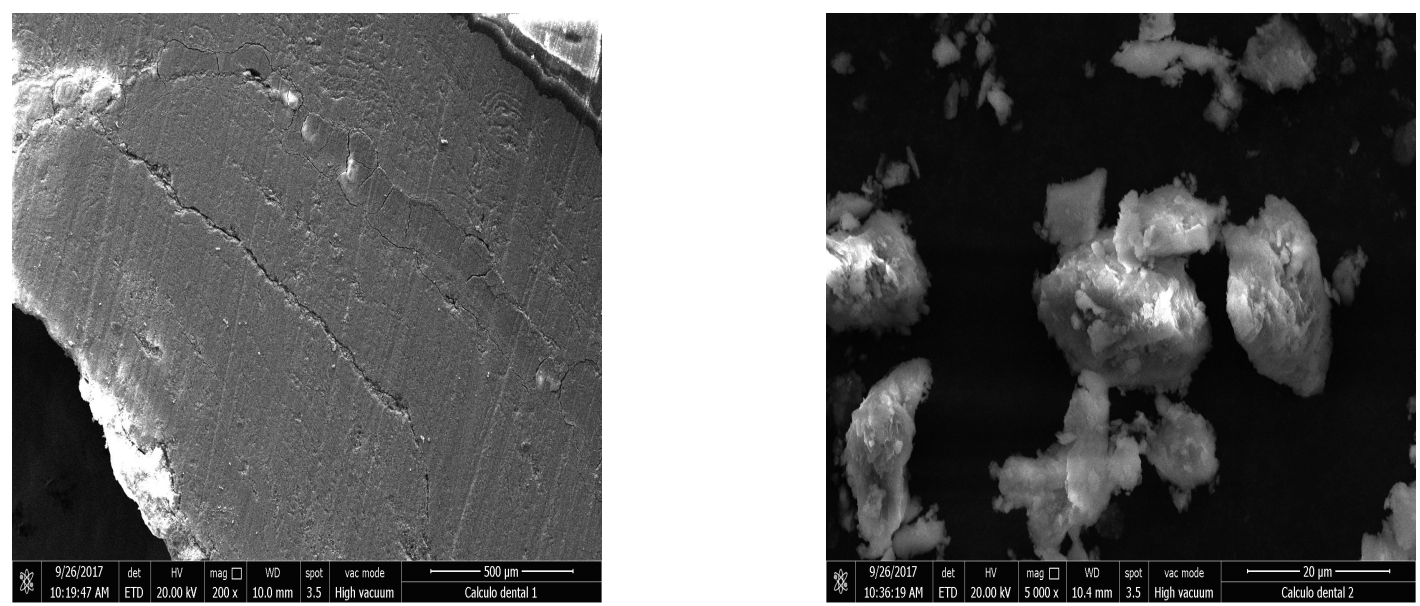

Figura 5: Imágenes obtenidas del microscopio electrónico de barrido, del sialolito y del cálculo respectivamente

intensidad, $\theta$ es el ángulo de difracción, obteniendo los siguientes resultados:

- $t(211)=36.40 \mathrm{~nm}$ para el cálculo dental.

- $t(211)=24.27 \mathrm{~nm}$ para el sialolito.

\section{| MICROSCOPÍA ELECTRÓNICA DE BARRIDO, ANÁLISIS POR EDX}

A las muestras se les realizó por Microscopía Electrónica de Barrido un análisis de EDX, para determinar cuáles son los componentes que constituyen las muestras, en la figura (5) se observa las imágenes obtenidas y exploradas con esta técnica, obteniendo los siguientes resultados:

El análisis por EDX mostró que las muestras tiene en mayor concentración los iones de fósforo y calcio, lo que nos indica que estos son fosfatos de calcio y puede ser observado en los espectros obtenidos y mostrados en las figura (6).

\section{MICROSCOPÍA DE FUERZA ATÓMICA.}

Se realizó la Microscopia de Fuerza Atómica a las muestras de cálculo y del sialolito, y en donde se evidencian cristales de hidroxiapatita que se van acumulando en capas a medida que pasa el tiempo en los dientes.

Es importante recalcar que en estudios previos donde se involucra la hidroxiapatita a diversos usos biomédicos se han observado la formación de estos cristales (Monsalve, 2011), y se muestran en las figuras (7) y (8). En estas micrografías que se obtuvieron se puede observar la forma de los cristales además de cómo están ordenados, este ordenamiento es en conformación de capas de minerales que se van colocando unos encima de otros, favoreciendo de esta forma el crecimiento del cristal.

En el caso del sialolito se muestran las imágenes obtenidas con el MFA, a distintos tamaños y en donde se evidencia la irregularidad de la superficie. Y que al igual que con el cálculo dental, y estos se van agrupando en capas a medida que va pasando el tiempo generando así el crecimiento del mismo en la 

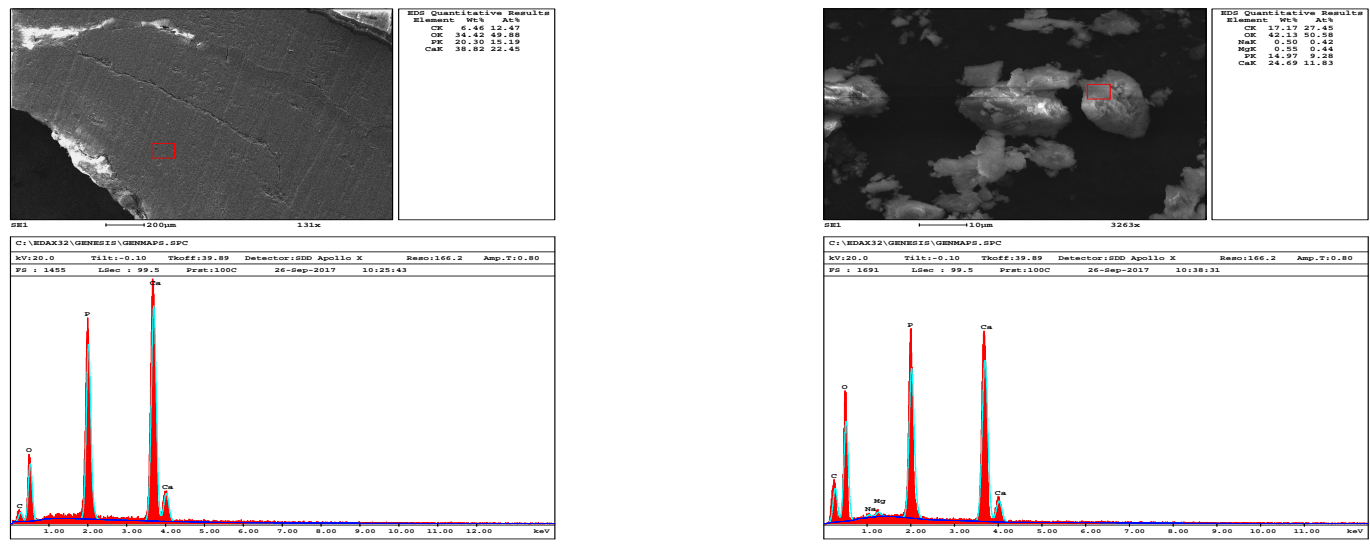

Figura 6: Espectro de EDX de las muestras de cálculo y de sialolito.
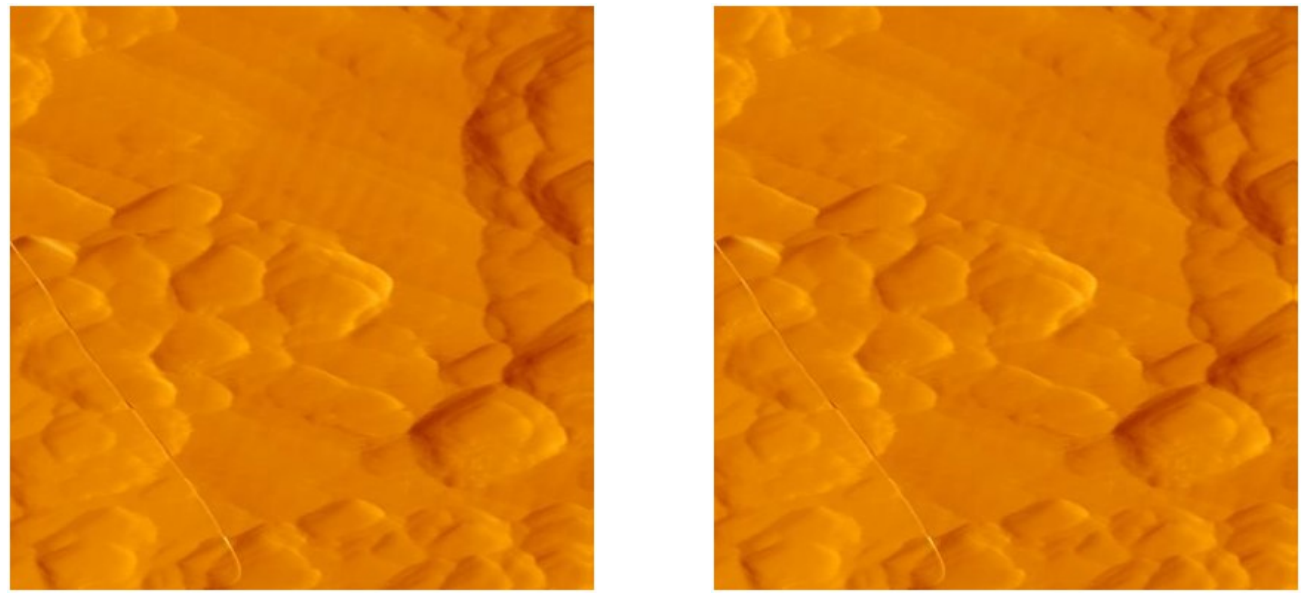

Figura 7: Imágenes de MFA de los cálculos dentales.

glándula, en las micrografías que se muestran a continuación se aprecian los tamaños y formas de los cristales de hidroxiapatita.

\section{| DISCUSIÓN}

En el análisis morfológico de las muestras con microscopía de fuerza atómica se puede observar que los cristales van creciendo en el medio en forma de capas unas sobre otras. De igual forma, el análisis realizado por difracción de rayos X y por EDX, muestra que el componente más predominante en los cálculos son los fosfatos de calcio, tales como la hidroxiapatita y que son los principales causantes de la formación de cálculo dental en los dientes y en los conductos salivales. 

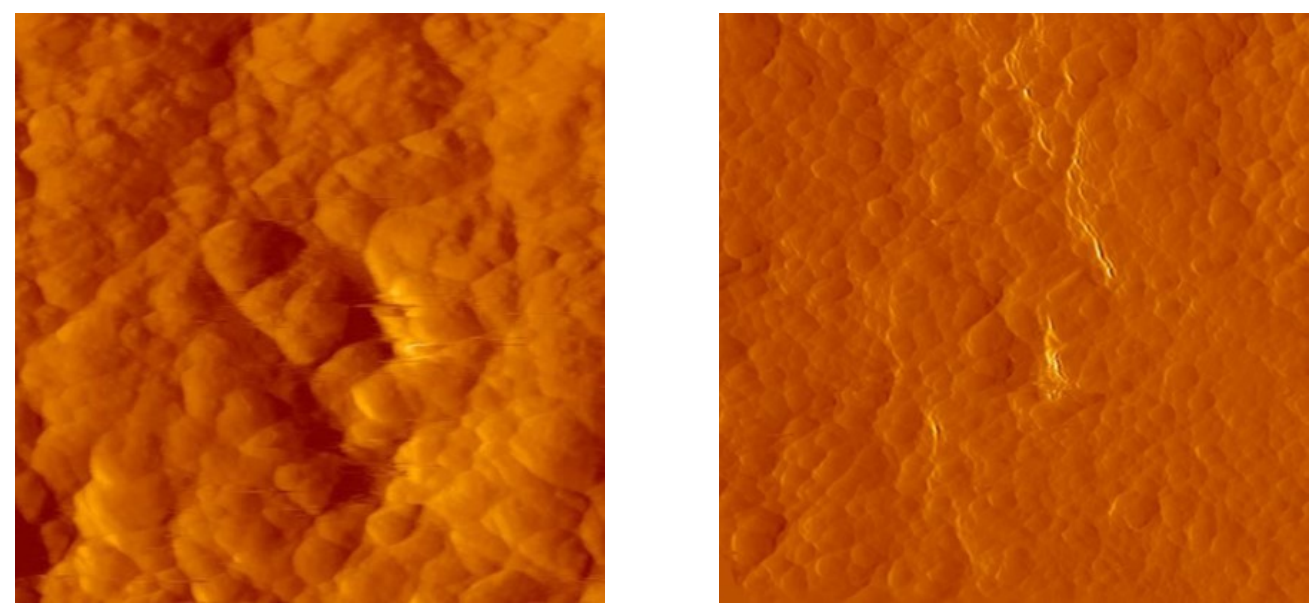

Figura 8: Imágenes de MFA del sialolito.

El tamaño de los cristales usando la fórmula de Scherer muestra que los cristales del sialolito son más pequeños, esto es debido a que los cristales tienden a compactarse más en estos puesto que la formación es más ordenada que en el caso de los cálculos.

\section{I CONCLUSIONES}

El análisis por difracción de rayos X de las muestras de cálculo dental y del sialolito encontró que la estructura que se forma en estos, es el que pertenece al grupo de los fosfatos de calcio, específicamente de la hidroxiapatita, calculando los parámetros de celda y los tamaños promedios de los cristales tanto de los cálculos dentales como del sialolito, usando como referencia el pico más intensoque se observa en el difractograma que es el que corresponde con los índices de Miller 211 y 002.

Por otro lado, a través del análisis por EDX que se realizó por medio de la microscopía electrónica de barrido se obtuvieron los compuestos de las muestras, observando que en su mayoría están constituidos por fosforo y calcio, confirmando de esta forma que estas muestras son fosfatos de calcio (Hidroxiapatita).

El crecimiento de cálculos dentales en la boca es un problema que involucra la salud bucal y entendiendo el mecanismo de formación de estos podemos controlar de mejor manera la formación de estos y todas las patologías que están relacionadas.

\section{REFERENCIAS}

A. J. Caballero, C. P., Ma. A. Fonseca. (2011). Cálculo dental una revisión de literatura y presentación de una condición inusual. Acta Odontológica Venezolana, 3(49), 1-10.

A. Loera, F. J. A., F. Y. Ramírez, y Guerrero, A. L. (2012). Biopelículas multiespecie: asociarse para sobrevivir. Invest Cienc, 54, 49-56.

B. Anuradha, e. a. (2016). Oral and salivary changes in patients with chronic kidney disease: A clinical and biochemical study. Journal of Indian Society of Periodontology(19), 297-301.

Cullity, B. (1959). Elements of x -ray diffraction. Addison Wesley, 297-323.

Dos Reis, D. e. a. (2016). Analysis of the early stage and evolution of dental enamel erosion. Brazilian Dental 
Journal(27), 313-317.

J. Hayashizaki, e. a. (2008). Site specific mineral composition and microstructure of human supra-gingival dental calculus. Arch Oral Biol(53), 168-74.

Monsalve, e. a. (2011). Caracterización por microscopía de fuerza atómica (mfa) y microscopía de barrido (meb) de recubrimientos de hidroxiapatita obtenidos por el método biomimético sobre acero inoxidable 3161. Acta Microscópica, 1(20), 45-53.

S. R. Epstein, I. W. S., I. Mandel. (1980). Salivary composition and calculus formation in patients undergoing hemodialysis. J Periodontol(51), 336-8.

White, D. (2000). Recent advances in methods for the assessment of dental calculus research and clinical implications. Monogr Oral Sci.(17), 163-73.

y M. E. González, S. B. L. (2016). La biopelícula: una nueva concepción de la placa dentobacteriana. Medicentro Electrónica(20), 167-175. 